\title{
Flexural Strength of Different Monolithic Computer-Assisted Design and Computer-Assisted Manufacturing Ceramic Materials upon Different Thermal Tempering Processes
}

\author{
Niwut Juntavee ${ }^{1}$ Pithiwat Uasuwan ${ }^{2}$ \\ ${ }^{1}$ Department of Prosthodontics, Faculty of Dentistry, Khon Kaen \\ University, Khon Kaen, Thailand \\ 2Division of Biomaterials and Prosthodontics Research, Faculty of \\ Dentistry, Khon Kaen University, Khon Kaen, Thailand
}

\begin{abstract}
Address for correspondence Niwut Juntavee, DDS, CAGS, MSD, DScD, Department of Prosthodontics, Faculty of Dentistry, Khon Kaen University, Khon Kaen 40002, Thailand (e-mail: niwutpapa@hotmail.com).
\end{abstract}

Eur J Dent:2020;14:566-574

\begin{abstract}
Keywords

- flexural strength

- lithium silicate

- lithium di-silicate

- monolithic zirconia

- thermal tempering

Objective Strength of ceramics related with sintering procedure. This study investigated the influence of different tempering processes on flexural strength of three monolithic ceramic materials.

Materials and Methods Specimens were prepared in bar-shape (width $\times$ length $\times$ thickness $=4 \times 14 \times 1.2 \mathrm{~mm}$ ) from yttria-stabilized tetragonal zirconia polycrystalline (Y-TZP, inCoris TZI [I]), zirconia-reinforced lithium silicate (ZLS, Vita Suprinity [V]), and lithium disilicate ( $\mathrm{LS}_{2}$, IPS e.max CAD [E]), and sintered with different tempering processes: slow (S), normal $(N)$, and fast $(F)$ cooling procedure $(n=15 /$ group). Flexural strength $(\sigma)$ was determined using three-point bending test apparatus at $1 \mathrm{~mm} / \mathrm{min}$ crosshead speed.

Statistical Analysis The analysis of variance and Bonferroni's multiple comparisons were determined for significant difference $(\alpha=0.05)$. Weibull analysis was applied for survival probability, Weibull modulus $(\mathrm{m})$, and characteristics strength $\left(\sigma_{o}\right)$. Microstructures were evaluated with scanning electron microscope and X-ray diffraction.

Results The mean \pm standard deviation (MPa) of $\sigma$, $\mathrm{m}$, and $\sigma_{\mathrm{o}}$ were: $1,183.98 \pm 204.26$, $6.23,1,271.80$ for IS; $1,084.43 \pm 204.79,5.76,1,170.08$ for IN; $777.19 \pm 99.77,8.78$, 819.96 for IF; $267.15 \pm 32.71,9.11,281.48$ for VS; $218.43 \pm 38.46,6.40,234.23$ for VN; $252.67 \pm 37.58,7.20,269.23$ for VF; $392.09 \pm 37.91,11.37,409.23$ for ES; $378.88 \pm 55.38,7.45,403.11$ for EN, and $390.94 \pm 25.34,16.00,403.51$ for EF. Thermal tempering significantly affected flexural strength of Y-TZP $(p<0.05)$, but not either ZLS or $\mathrm{LS}_{2}(p>0.05)$. Y-TZP indicated significantly higher flexural strength upon slow tempering than others.

Conclusion Enhancing flexural strength of Y-TZP can be achieved through slow tempering process and was suggested as a process for monolithic zirconia. Strengthening of ZLS and $\mathrm{LS}_{2}$ cannot be accomplished through tempering; thus, either $\mathrm{S}-, \mathrm{N}$-, or $\mathrm{F}$ tempering procedure can be performed. Nevertheless, to minimize sintering time, rapid thermal tempering is more preferable for both $\mathrm{ZLS}$ and $\mathrm{LS}_{2}$.
\end{abstract}

DOI https://doi.org/

$10.1055 / \mathrm{s}-0040-1713957$

ISSN 1305-7456.
(C2020 Dental Investigation Society
License terms

() (1) $\ominus \circledast$ 


\section{Introduction}

The demands of patients regarding esthetics, as well as the development of technology, have convinced most clinicians to use materials that possess the extraordinary optical properties to replicate the color of natural dentition and the ability to withstand heavy occlusal force. ${ }^{1}$ As such, ceramics are advocated as the restorative materials in conjunction with their biocompatibility. ${ }^{2}$ Over the last three decades, there was a rapid expansion of ceramic use, leading to a variety of new ceramic materials. Lithium disilicate $\left(\mathrm{LS}_{2}\right)$ glass-ceramics are forefront ceramic materials used for fixed dental prostheses, which are ideal for the restorations in aesthetic zone. ${ }^{3,4}$ To increase the strength of $\mathrm{LS}_{2}$ glass-ceramics, more zirconia was added, then called zirconia-reinforced lithium silicate (ZLS) glass-ceramics, and this was advocated for use in the heavier occlusal force area over original $\mathrm{LS}_{2}$ glass-ceramics. This new material not only supported the esthetic demands of the patients, but also contained the strength to resist occlusal force as in posterior restoration. Currently, zirconia is used to construct many dental restorations, such as posterior crowns and long-span bridges, as well as implant components, due to its exceptional strength. ${ }^{5}$ The zirconia microstructures comprise three crystalline phases of monoclinic $(m)$, tetragonal $(t)$, and cubic (c) with glass content. The presence of stable $\mathrm{m}$-phase is at the room temperature till reaching $1,170^{\circ} \mathrm{C}$, then the t-phase exists, and changes to the c-phase at $2,370^{\circ} \mathrm{C}$. During temperature cooling, a transformation from the $\mathrm{t}$ - to $\mathrm{m}$-phase occurs, resulting in a volumetric expansion of 3 to $5 \%$. This expansion phenomenon exerts compressive stresses at the crack tip and eventually interrupts the propagation of the crack. ${ }^{6}$ By adding a stabilizing agent for example, yttrium oxide, zirconia can be stabilized at a $t$-phase as in room temperature. ${ }^{7}$ The ability to inhibit the crack propagation-so-called "transformation toughening"is known as the strongest benefit of yttria-stabilized tetragonal zirconia polycrystalline (Y-TZP) ceramics. ${ }^{8,9}$

Zirconia ceramic loses strength as the grain size enlarges due to its susceptibility to spontaneous t- to m-phase transformation. ${ }^{10}$ To simulate natural tooth appearance, low fusing porcelain was veneered over zirconia substructure to remedy its opacity and dull appearance; however, chipping or deveneering of ceramic was a serious disadvantage. ${ }^{11-13}$ Thus, monolithic zirconia was developed to overcome the crack of the material's interface by fabricating a single material for an entire restoration. Computer-assisted design and computerassisted manufacturing (CAD-CAM) was used to construct the zirconia restoration. This can be applied through two different methods. One involved the use of presintered block to generate an oversized material framework and then sintered, resulting in a 20 to $25 \%$ shrinkage from its original dimension to achieve the final dimension. The alternative technique utilized fully sintered block for milling to derive for final dimension of restoration with no further sintering process. ${ }^{14}$ Nevertheless, the dental ceramic materials need to be sufficiently durable for clinical performance. ${ }^{15}$ Flexural strength is an essential and plausible method, which is widely used to assess the durability of ceramic materials. The restorations with high flexural strength, together with structural reliability indicate less possibility of fracturing. ${ }^{16,17}$

The sintering procedure was involved in the fabrication of the restorations, particularly in the approach using presintered blocks after milling. ${ }^{18}$ The sintering process is mainly categorized into three phases: a heating phase, a sintered-holding phase at the peak sintering temperature, and a cooling phase. ${ }^{19}$ The sintering procedure can be altered to optimize the mechanical and optical properties of zirconia and other ceramic materials. To achieve a completely sintered zirconia, the temperature from the sintering furnace was initially transmitted to the surface of the restoration and was eventually distributed to the material core by thermal conduction. Some technical alterations in sintering procedures were introduced to promote for better mechanical and optical properties of ceramics. ${ }^{20}$ The crystalline structure and content were directly impacted by the sintering parameters. ${ }^{21-23}$ Alterations in the sintered-holding time of the sintering procedure may have affected the grain growth and size, thus possibly having an impact on the strength and translucency of zirconia. ${ }^{24-26} \mathrm{~A}$ previous study has illustrated that increasing the sintered temperature of zirconia can result in better translucency with a little effect on strength. ${ }^{1}$ Another study has shown that an increased sintering temperature and a prolonged sintered holding time lead to raising the flexural strength of zirconia. ${ }^{27}$

The effects of heat rates to sintering temperature and sintered holding times on flexural strength have been reported, while the information regarding the effects of cooling procedure which directly relate with thermal tempering of ceramic material are unavailable. As such, this study aimed at the investigation the effects of thermal tempering on flexural strength of monolithic ceramic materials. The null hypotheses were that either different ceramic materials or varying thermal tempering processes or their combinations would not induce any influence on flexural strength of monolithic ceramic materials.

\section{Materials and Methods}

\section{Preparation the Specimens}

Three monolithic ceramic blocks of shade A2 as presented in - Table 1-partially sintered Y-TZP (inCoris TZI [I], Sirona, Bensheim, Germany), ZLS (Vita Suprinity [V]; Vita, Bad Säckingen, Germany), and $\mathrm{LS}_{2}$ (IPS e.max CAD [E]; IvoclarVivadent, Schaan, Liechtenstein)-were sectioned into the bar shapes ( $n=45$ for each type) using diamond coating blade (Isomet 1000; Beuhler, Lake Buff, Illinois, United States). The ZLS and $\mathrm{LS}_{2}$ bars were ground flat with SiC-abrasive paper 200 till 1,200, and polished with $1 \mu \mathrm{m}$ diamond suspension using a polishing machine (Ecomet 3; Beuhler, Lake Bluff, Illinois, United States) to derive for the expected dimensions (width $\times$ length $\times$ thickness $=4 \times 14 \times 1.2 \mathrm{~mm}$ ). The Y-TZP specimens were prepared in oversized dimensions (width $\times$ length $\times$ thickness $=5 \times 17.5 \times 1.5 \mathrm{~mm}$ ) for compensation upon $20 \%$ sintering shrinkage. The bars were cleaned with 
the distilled water and dried for 60 minutes at normal room temperature.

Each type of ceramic bar was randomly divided into three subgroups ( $n=15$ ) based on the different thermal tempering processes with slow cooling $\left(5^{\circ} \mathrm{C} / \mathrm{min}[\mathrm{S}]\right)$, normal cooling $\left(25^{\circ} \mathrm{C} / \mathrm{min}[\mathrm{N}]\right)$, and fast cooling $\left(50^{\circ} \mathrm{C} / \mathrm{min}[\mathrm{F}]\right)$. One furnace (inFireHTC; Sirona, Bensheim, Germany) was programmed for I-specimens, and another furnace (Programat P310; Ivoclar-Vivadent, Schaan, Leichtenstein) was programmed for $\mathrm{V}$ - and E-specimen. The sintering program of I-specimen involved heating at $25^{\circ} \mathrm{C} / \mathrm{min}$ to $800^{\circ} \mathrm{C}$, then $15^{\circ} \mathrm{C} / \mathrm{min}$ to $1,510^{\circ} \mathrm{C}$, and finally cooling based on different thermal tempering processes. For $\mathrm{V}$-specimen, the materials were heated at $55^{\circ} \mathrm{C} / \mathrm{min}$ to $840^{\circ} \mathrm{C}$ and then each thermal tempering protocol was performed. For E-specimen, materials were heated at $60^{\circ} \mathrm{C} / \mathrm{min}$ to $770^{\circ} \mathrm{C}$, then $30^{\circ} \mathrm{C} / \mathrm{min}$ to $850^{\circ} \mathrm{C}$, followed by conducting the various thermal tempering protocols.

\section{Determination the Flexural Strength}

All specimens were evaluated for flexural strength in a universal testing machine (LR30/k; Lloyd, Leicester, England). Each specimen was aligned horizontally on a three-point bending apparatus (ISO 6872:2015) which consists of two supporting bars at $12 \mathrm{~mm}$ (L) apart from each other, and one vertically movable bar located between the supporting components as shown in - Fig. 1. The specimen was subjected to compressive load of the vertical bar with $1.0 \mathrm{~mm} / \mathrm{min}$ crosshead speed until the specimen fractured. The fracture load was recorded and calculated for flexural strength by Eq. 1 .

$$
\sigma=3 N L / 2 b d^{2}
$$

Table 1 Type, company, batch number, and chemical composition (\% by weight) of ceramic materials used in this study

\begin{tabular}{|l|l|l|l|l|}
\hline Material & Type & Company & Batch number & Composition \\
\hline $\begin{array}{l}\text { I: inCoris TZI } \\
\text { (C mono L A2) }\end{array}$ & $\begin{array}{l}\text { Yttria-stabilized tetragonal } \\
\text { zirconia polycrystalline }\end{array}$ & Sirona, Germany & 2017082019 & $\begin{array}{l}\geq 99.0 \% \mathrm{ZrO}_{2}+\mathrm{HfO}_{2}+\mathrm{Y}_{2} \mathrm{O}_{3}, 4.5-6.0 \% \\
\mathrm{Y}_{2} \mathrm{O}_{3}, \leq 5 \% \mathrm{HfO}_{2}, \leq 0.5 \% \mathrm{Al}_{2} \mathrm{O}_{3}, \leq 0.5 \% \mathrm{Al}_{2} \mathrm{O}_{3}\end{array}$ \\
\hline $\begin{array}{l}\mathrm{V}: \text { Vita Suprinity } \\
(\text { A2-HT LS-14) }\end{array}$ & $\begin{array}{l}\text { Zirconia-reinforced lithium } \\
\text { silicate glass }\end{array}$ & VITA, Germany & 42902 & $56-64 \% \mathrm{SiO}_{2}, 8-12 \% \mathrm{ZrO}_{2}, 15-21 \% \mathrm{Li}_{2} \mathrm{O}$ \\
\hline $\begin{array}{l}\text { E: IPS e.maxCAD } \\
\text { (HT A2/ C14) }\end{array}$ & $\begin{array}{l}\text { Lithium disilicate glass } \\
\text { ceramics }\end{array}$ & $\begin{array}{l}\text { Ivoclar Vivadent, } \\
\text { Liechtenstein }\end{array}$ & $\mathrm{X} 13380$ & $\begin{array}{l}57-80 \% \mathrm{SiO}_{2}, 11-19 \% \mathrm{Li}_{2} \mathrm{O},<13 \% \mathrm{~K}_{2} \mathrm{O}, \\
<11 \% \mathrm{P}_{2} \mathrm{O}_{5},<8 \% \mathrm{ZrO}_{2},<8 \% \mathrm{ZnO}\end{array}$ \\
\hline
\end{tabular}
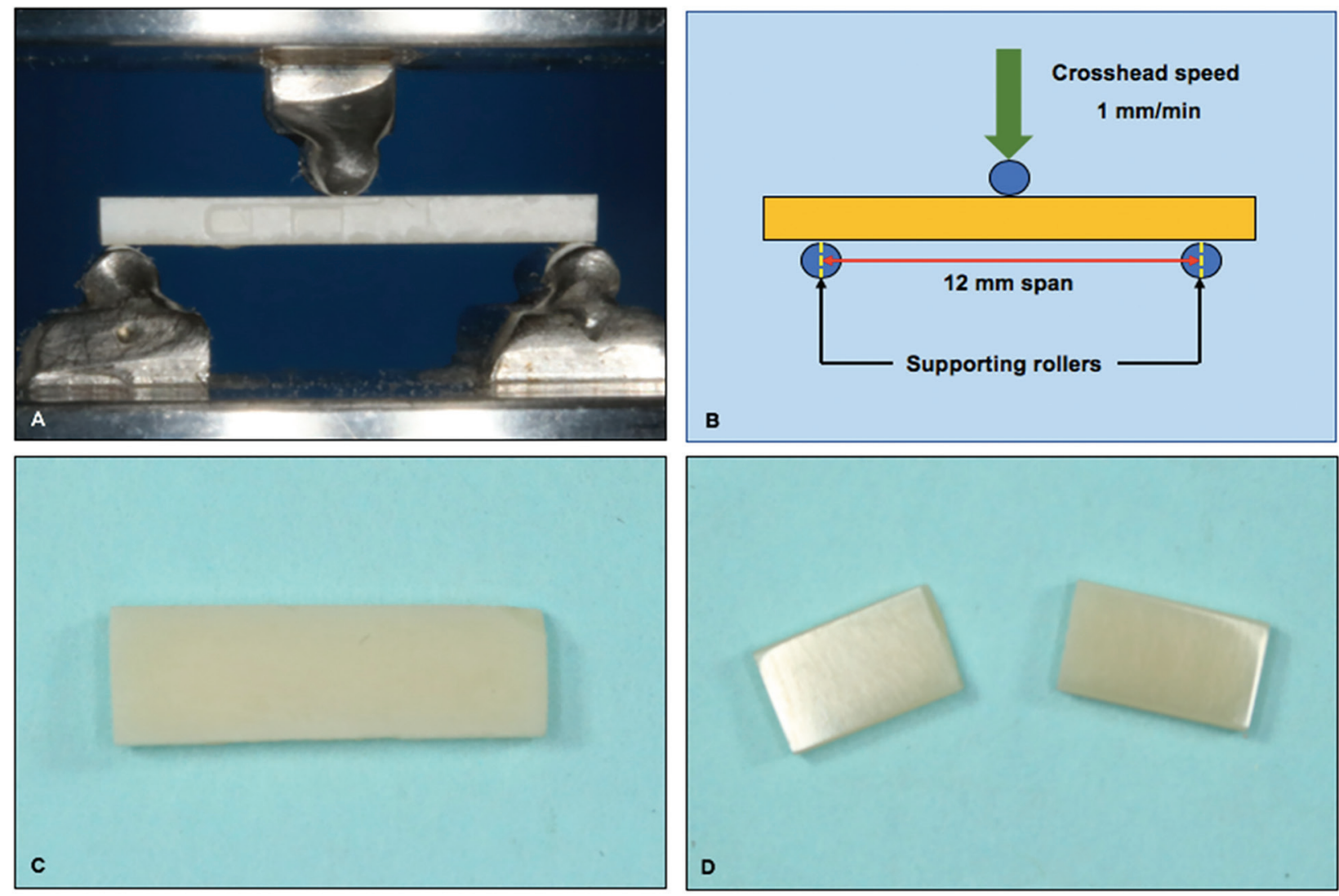

Fig. 1 Three-point bending test apparatus (A), schematic drawing of three-point bending test (B), test specimen (C), and fracture of specimen after three-point bending test (D). 
In which: $\sigma$ : flexural strength (MPa); $N$ : load at fracture (Newton); L: space between supporting bars ( $\mathrm{mm}$ ); $b$ : width of specimen ( $\mathrm{mm}$ ), and $d$ : thickness of specimen $(\mathrm{mm})$.

\section{Microstructure Evaluation}

The samples for group V and group E were etched with 5\% hydrofluoric acid for 1 minute to remove glassy structure. All samples were gold coated (10 mA of current, $130 \mathrm{~m}$-torr of vacuum) for 3 minutes and dried in a desiccator. The topographic surfaces and fracture characteristics were examined with scanning electron microscope (SEM, Hitachi, Osaka, Japan). The crystalline structures of materials were evaluated for the proportions of their crystal structures using an X-ray diffraction (XRD, PANalytical, Empyrean, Almelo, Netherlands) by scanning at a diffraction angle $(2 \theta)$ of 20 to 40 degrees, with step size of 0.02 degrees at 2 seconds interval utilizing copper $\mathrm{k}-\alpha(\mathrm{Cu} \mathrm{k}-\alpha)$ beam. The phase was analyzed by match referencing with the standard reference database. The $\mathrm{m}$ - to $\mathrm{t}$-phase proportion was determined from the peak intensities using software X'Pert-Plus (Philips; Almelo, Netherlands) and calculated for the $\mathrm{m}$ - phase as a fraction of the entire phase $\left(\mathrm{X}_{\mathrm{m}}\right)$ with Garvie-Nicholson formula, as shown in Eq. 2 to $4 .{ }^{28}$

$$
X_{m}=\frac{I_{m}(111)+I_{m}(11 \overline{1})}{I_{m}(111)+I_{m}(11 \overline{1})+I_{t}(111)}
$$

Where $\mathrm{I}_{\mathrm{m}}$ and $\mathrm{I}_{\mathrm{t}}$ refer to the integrated intensities of the $\mathrm{m}$ - and t-phases, respectively.

$$
\mathrm{X}_{\mathrm{m}}=\frac{\mathrm{CX}}{1+(\mathrm{C}-1) \mathrm{X}_{\mathrm{m}}}
$$

Where $\mathrm{C}$ refers to compositional-dependent corrected factor $(C=1.32)$.

$$
\mathrm{X}_{\mathrm{t}}=1-\mathrm{X}_{\mathrm{m}}
$$

Where $\mathrm{X}_{\mathrm{t}}$ refers to the Toraya-corrected mass fraction of $t$-phase

\section{Statistical Analysis}

Analysis of variance (ANOVA) and post hoc Bonferroni multiple comparisons, using SPSS-Win20 (IBM; Chicago, Illinois, United States), were performed for the significant differences of flexural strength for monolithic materials when sintered at different thermal tempering processes. Results were considered statistically significant at $p<0.05$. The grain size and phase composition were relatively compared. Weibull analysis was performed to determine the reliability of strength by using Weibull++ statistics (ReliaSoft; Tucson, Arizona, United States) and Weibull modulus ( $\mathrm{m})$.

\section{Results}

The mean, standard deviation (SD), and 95\% confidence interval of flexural strength, Weibull modulus $(\mathrm{m})$, and characteristic strength $\left(\sigma_{0}\right)$ were illustrated in - Table 2 and - Fig. 2. The mean \pm SD values of flexural strength (MPa) were: $1,183.98 \pm 204.26$ for IS; $1,084.43 \pm 204.79$ for IN; $777.19 \pm 99.77$ for IF; $267.15 \pm 32.71$ for VS; $218.43 \pm 38.46$ for VN; $252.67 \pm 37.58$ for VF; $392.09 \pm 37.91$ for ES; $378.88 \pm$ 55.38 for EN, and $390.94 \pm 25.34$ for EF. The highest flexural strength was demonstrated ingroup IS, followed by IN, IF, ES, $\mathrm{EF}, \mathrm{EN}, \mathrm{VS}, \mathrm{VF}$, and VN. The evaluated results of the characteristic strength $\left(\sigma_{0}, \mathrm{MPa}\right)$ for IS, IN, IF, VS, VN, VF, ES, EN, and EF were 1,271.80, 1,170.08, 819.96, 281.48, 234.23, 269.23, 409.23, 403.11, and 403.51, respectively. The mean \pm SD values of flexural strength (MPa) of $\mathrm{I}, \mathrm{V}$, and $\mathrm{E}$ groups were $1,015.2 \pm 245.86,246.08 \pm 41.08$, and $387.31 \pm 40.91$, respectively. The mean \pm SD values of flexural strength (MPa) of the $\mathrm{S}, \mathrm{N}$, and F groups were $614.41 \pm 427.35,560.58 \pm 399.39$, and $473.60 \pm 232.83$, respectively.

ANOVA revealed significant different flexural strength upon ceramic materials, thermal tempering process, and their interactions $(p<0.05)$ as shown in -Table 3. Post hoc Bonferroni multiple comparisons indicated significant different in flexural strength among groups of ceramic materials $(p<0.05)$ as presented in - Table 4 and - Fig. 2. Significant different flexural strength as a result of thermal tempering

Table 2 Mean, standard deviation, 95\% confidential interval of flexural strength (MPa), Weibull modulus (m), characteristic

\begin{tabular}{|c|c|c|c|c|c|c|c|c|c|}
\hline \multirow[t]{2}{*}{ Group } & \multirow[t]{2}{*}{$n$} & \multirow{2}{*}{$\begin{array}{l}\text { Flexural strength } \\
\text { Mean } \pm \text { SD }\end{array}$} & \multicolumn{2}{|l|}{$95 \% \mathrm{Cl}$} & \multirow[t]{2}{*}{$\mathrm{m}$} & \multirow[t]{2}{*}{$\sigma_{0}$} & \multicolumn{2}{|c|}{ Relative phase } & \multirow{2}{*}{$\begin{array}{l}\text { Average } \\
\text { grain size }\end{array}$} \\
\hline & & & LB & UB & & & m-phase & t-phase & \\
\hline IS & 15 & $1,183.98 \pm 204.26$ & $1,070.86$ & $1,297.09$ & 6.23 & $1,271.80$ & 0.0731 & 0.9269 & 0.460 \\
\hline IN & 15 & $1,084.43 \pm 204.79$ & 971.02 & $1,197.85$ & 5.76 & $1,170.08$ & 0.0765 & 0.9235 & 0.470 \\
\hline IF & 15 & $777.19 \pm 99.77$ & 721.94 & 832.44 & 8.78 & 819.96 & 0.0817 & 0.9183 & 0.482 \\
\hline VS & 15 & $267.15 \pm 32.71$ & 249.04 & 285.27 & 9.11 & 281.48 & 0 & 1 & 0.873 \\
\hline VN & 15 & $218.43 \pm 38.46$ & 197.13 & 239.73 & 6.40 & 234.23 & 0 & 1 & 0.601 \\
\hline VF & 15 & $252.67 \pm 37.58$ & 231.86 & 273.48 & 7.20 & 269.23 & 0 & 1 & 0.609 \\
\hline ES & 15 & $392.09 \pm 37.91$ & 371.10 & 413.09 & 11.37 & 409.23 & & & 1.724 \\
\hline EN & 15 & $378.88 \pm 55.38$ & 348.22 & 409.55 & 7.45 & 403.11 & & & 1.707 \\
\hline EF & 15 & $390.94 \pm 25.34$ & 376.91 & 404.97 & 16.00 & 403.51 & & & 1.664 \\
\hline
\end{tabular}
strength $\left(\sigma_{0}\right)$, relative monolithic (m-) and tetragonal (t-) phase content (weight \%), and average grain size ( $\left.\mu \mathrm{m}\right)$ of inCoris TZI (I), Vita Suprinity (V), IPS e.max CAD (E) upon slow (S), normal (N), and fast (F) thermal tempering processes

Abbreviations: $\mathrm{Cl}$, confidential interval; df, degree of freedom; F, F-ratio; MS, mean square; $\mathrm{p}, \mathrm{p}$-value; SD, standard deviation; SS, sum of squares. 

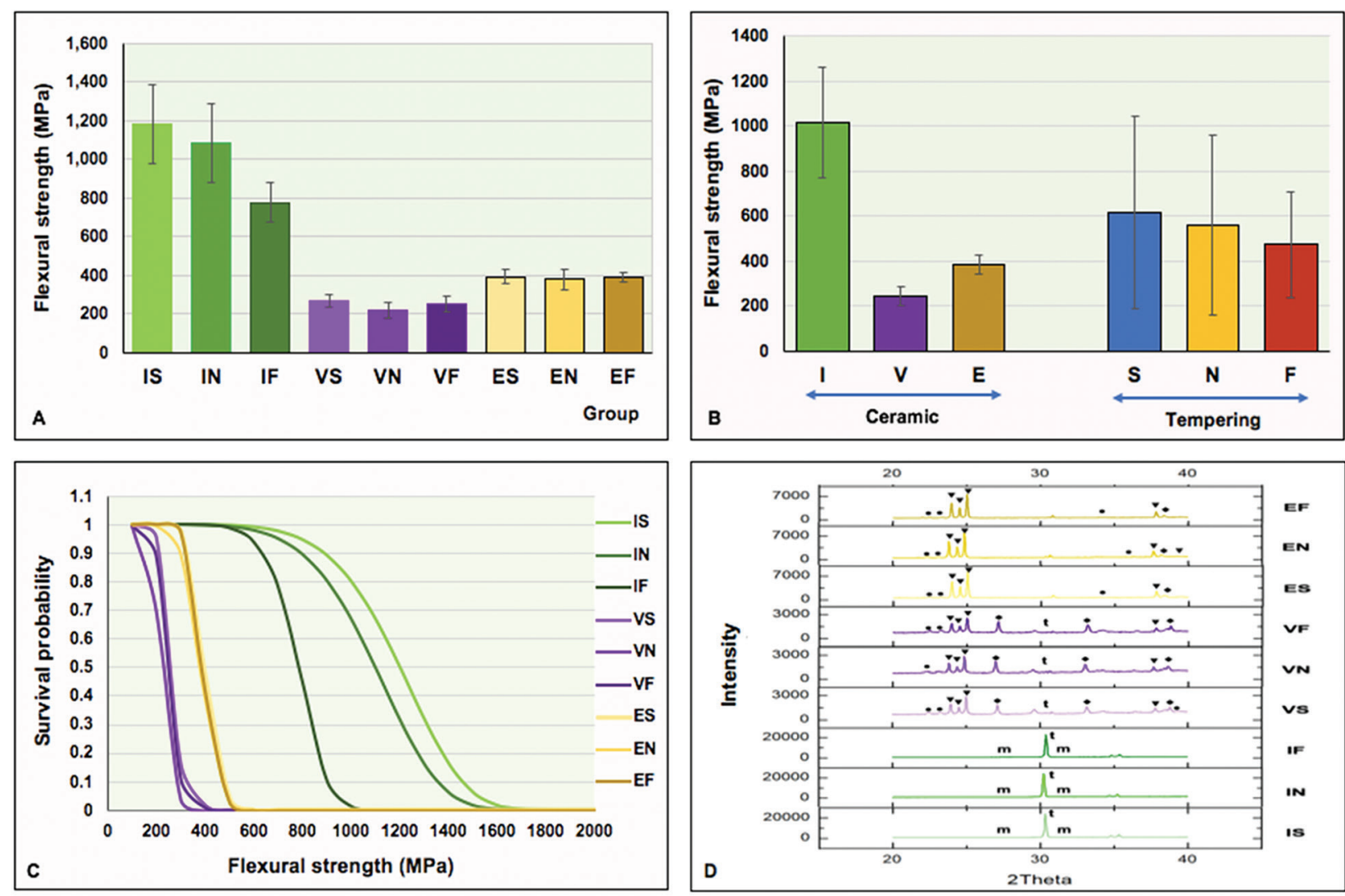

Fig. 2 Flexural strength (A, B), Weibull analysis (C), and X-ray diffraction pattern (D) of inCoris TZI (I), Vita Suprinity (V), IPS e.max CAD (E) upon slow $(\mathrm{S})$, normal $(\mathrm{N})$, and fast $(\mathrm{F})$ thermal tempering processes.

Table 3 An analysis of variance of flexural strength of three monolithic ceramic materials upon different thermal tempering processes

\begin{tabular}{|l|l|l|l|l|l|}
\hline Source & SS & df & MS & F test & $p$-Value \\
\hline Corrected model & $16,455,356.729$ & 8 & $2,056,919.591$ & 180.212 & 0.000 \\
\hline Intercept & $40,767,560.220$ & 1 & $40,767,560.220$ & $3,571.750$ & 0.000 \\
\hline Material & $15,086,049.464$ & 2 & $7,543,024.732$ & 660.864 & 0.000 \\
\hline Tempering process & $454,350.451$ & 2 & $227,175.226$ & 19.903 & 0.000 \\
\hline Material $\times$ tempering process & $914,956.814$ & 4 & $228,739.204$ & 20.040 & 0.000 \\
\hline Error & $1,438,150.014$ & 126 & $11,413.889$ & & \\
\hline Total & $58,661,066.964$ & 135 & & & \\
\hline
\end{tabular}

Abbreviations: df, degree of freedom; F, F-ratio; LB, lower bound; MS, mean square; p, p-value; SS, sum of squares; UB, upper bound.

also indicated among groups ( $p<0.05)$, except for S-N as presented in $\boldsymbol{\sim}$ Table $\mathbf{4}$ and $\boldsymbol{\sim}$ Fig. $\mathbf{2}$. The interaction of monolithic materials and thermal tempering processes indicated significant impact on flexural strength $(p<0.05)$, except for IS-IN, VS-VN, VS-VF, VS-ES, VS-EN, VS-EF, VN-VF, VF-EN, ES-EN, ES-EF, and EN-EF as presented in - Table 4 and - Fig. 2. Weibull analysis of flexural strength values illustrated the Weibull modulus from highest to lowest as: EF (16.00), ES (11.37), VS (9.11), IF (8.78), EN (7.45), VF (7.20), VN (6.40), IS (6.23), and IN (5.76). These values indicated the relative survival probability of flexural strength as shown in - Table 4 and - Fig. 2 .
The microscopic structures of I-, V-, and E-samples at different thermal tempering processes, and the fractographic micrograph of fracture surfaces for tested groups were presented in $\boldsymbol{- F i g}$. 3. For group-I, the crystalline structures were composed of small grain size $(0.1-0.38 \mu \mathrm{m})$, medium grain size $(0.39-0.66 \mu \mathrm{m})$, and large grain size $(0.67-0.93 \mu \mathrm{m})$ but were predominately medium grain. Fast thermal tempering was capable of initiating grain growth, which illustrated as increasing the medium and large grain, more efficient than either slow or normal tempering protocols. The grain sizes $(\mu \mathrm{m})$ were approximately 0.460 for IS, 0.470 for IN, and 0.482 for IF. The SEM analysis for the slow and normal thermal 
Table 4 Post hoc Bonferroni multiple comparisons of flexural strength of inCoris TZI (I), Vita Suprinity (V), IPS e.max CAD (E) upon slow $(\mathrm{S})$, normal $(\mathrm{N})$, and fast $(\mathrm{F})$ thermal tempering processes

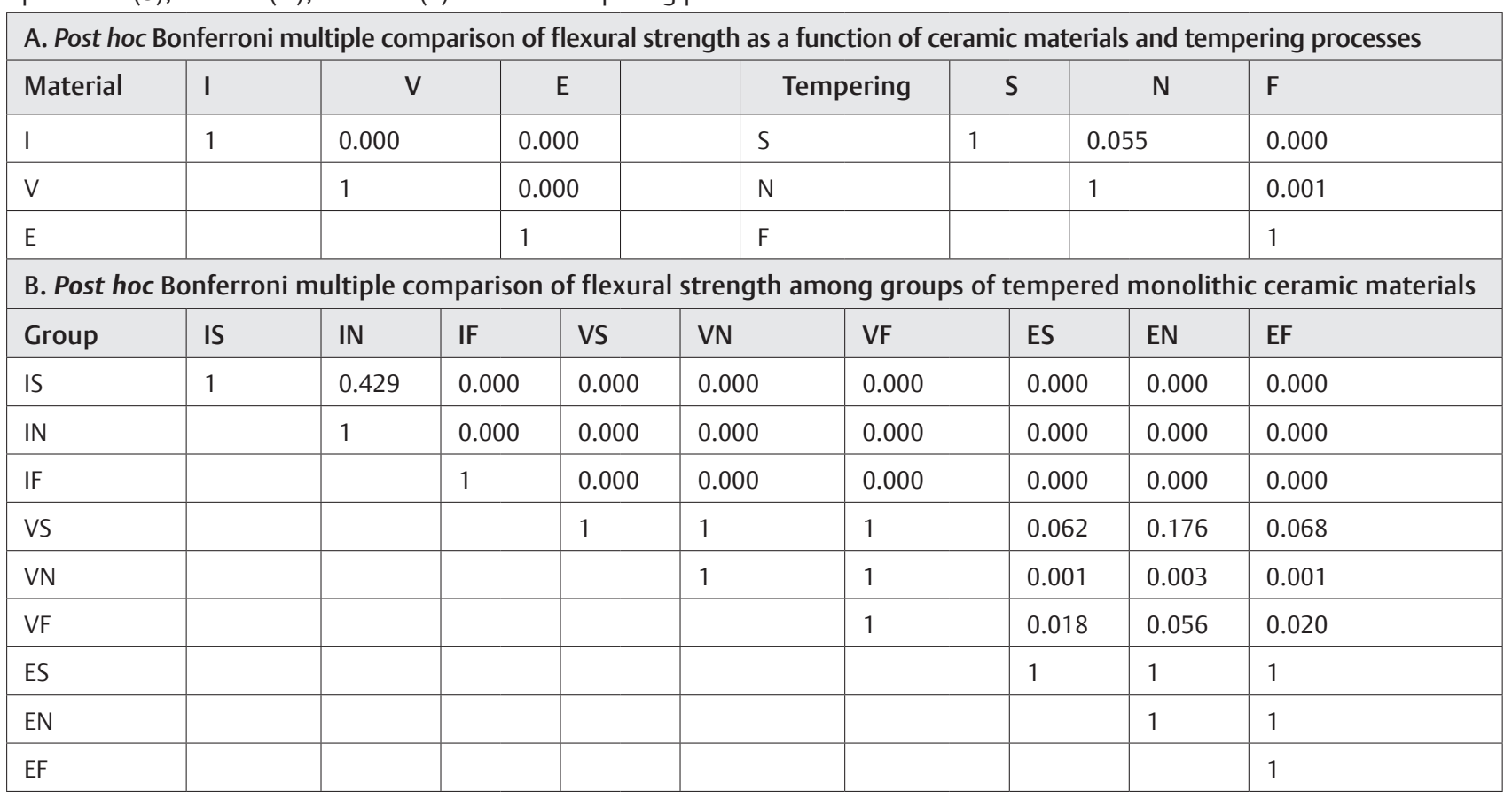

tempering groups depicted crystal structures with defective integration at the grain boundaries. The V-groups showed a minute space shuttle-like crystalline structure of $\mathrm{LS}_{2}$, and zirconia grains as observed in the micrograph shown in - Fig. 3 . The crystalline structures for group-VN and group-VF were more packing and less porosities compared with the group-VS. The $\mathrm{LS}_{2}$ grain sizes $(\mu \mathrm{m})$ were approximately were 0.873 for $\mathrm{VM}, 0.601$ for $\mathrm{VN}$, and 0.609 for VF as presented in - Table 2. The SEM analysis of E-groups illustrated a minute space shuttle-like crystalline structure of $\mathrm{LS}_{2}$. Likewise, the microstructure of ES showed a higher density of grain with less porosity compared with EN and EF as shown in - Fig. 3. The $\mathrm{LS}_{2}$ grain sizes $(\mu \mathrm{m})$ were approximately 1.724 for ES, 1.707 for EN, and 1.664 for EF as presented in - Table 2.

The crystalline contents and phases of specimens analyzed by XRD as shown in - Table 2 and - Fig. 3. The XRD patterns of I-group showed a large proportion of tetragonal crystal structures with little proportion of monoclinic. The t-phases were presented at $2 \theta$ angle of $30.20,34.63$, and 35.20 degrees for IS and IN; and at 30.11, 34.53, and 35.09 degrees for IF-group. The $\mathrm{m}$-phases exhibited at $2 \theta$ angle of 27.79 and 31.12 degrees for groups-I. The crystalline patterns were coincided with the standard crystallographic database. The relative weight percentage (wt. \%) for $\mathrm{m}$-phases to the entire amount of zirconia phases varied in association with the amount of $\mathrm{t}$ - to $\mathrm{m}$-phase transformation, owing to firing parameters, as presented in - Table $\mathbf{2}$. The relative concentrations (wt. \%) of the m-/t-phases were: $0.0731 / 0.9269$ for IS; $0.0765 / 0.9235$ for IN; and $0.0817 / 0.9183$ for IF. The phase proportions were associated with thermal tempering. Higher increasing in the amount of m-crystal structure was indicated upon fast thermal tempering process.
The XRD pattern for group-V illustrated a large amount of $\mathrm{LS}_{2}$ crystal structures, followed by lithium metasilicate, lithium orthophosphate, and tetragonal phase of zirconia. The VS-group presented with $\mathrm{LS}_{2}$ crystal at $2 \theta$ angle of 23.94 , 24.51, 25.00, and 37.81 degrees while detecting lithium metasilicate crystal at $2 \theta$ angle of $27.11,33.17$, and 38.39 degrees; representing lithium orthophosphate crystal at $2 \theta$ angle of 22.37, 23.24, and 38.80 degrees; and also observing tetragonal zirconia at $2 \theta$ angle of 30.29 degrees. The VS-group presented with $\mathrm{LS}_{2}$ crystal at $2 \theta$ angle of 23.80 , $24.36,24.85$, and 37.67 degrees while detecting lithium metasilicate at $2 \theta$ angle of $26.96,33.04$, and 38.41 degrees; representing lithium orthophosphate at $2 \theta$ angle of 22.31 degrees; and also observing tetragonal zirconia at $2 \theta$ angle of 30.14 degrees. The VF-group was presented with $\mathrm{LS}_{2}$ crystal at $2 \theta$ angle of $23.96,24.52,25.02$, and 37.83 degrees while detecting lithium metasilicate at $2 \theta$ angle of $27.13,33.20$, and 38.58 degrees; representing lithium orthophosphate at $2 \theta$ angle of 22.31 and 23.15 degrees; and also observing tetragonal zirconia at $2 \theta$ angle of 30.24 degrees.

The XRD pattern for group-E indicated the great amount of $\mathrm{LS}_{2}$ and lithium metasilicate crystal, with minimal amount of lithium orthophosphate. The ES-group presented with $\mathrm{LS}_{2}$ crystal at $2 \theta$ angle of $23.97,24.53,25.03$, and 37.83 degrees while detecting lithium metasilicate at $2 \theta$ angle of 38.37 degrees and representing lithium orthophosphate at $2 \theta$ angle of 22.51, 23.26, and 34.02 degrees. The EN-group was presented with $\mathrm{LS}_{2}$ crystal at $2 \theta$ angle of $23.80,24.37,24.86$, 37.67 , and 39.30 degrees while detecting lithium metasilicate at $2 \theta$ angle of 38.21 degrees and representing lithium orthophosphate at $2 \theta$ angle of 22.34, 23.11, and 36.39 degrees. The EF-group presented with $\mathrm{LS}_{2}$ crystal at $2 \theta$ angle 

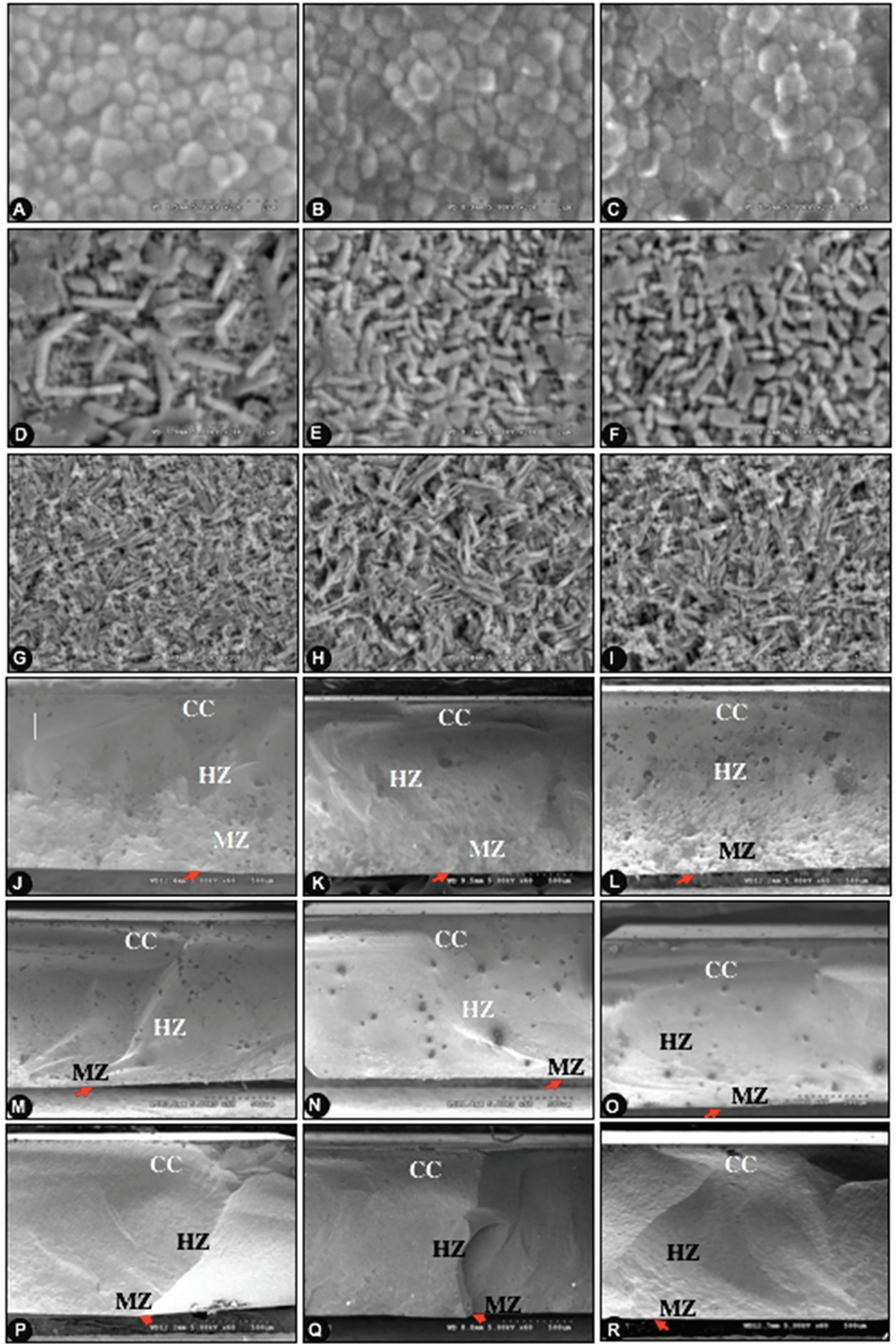

Fig. 3 Scanning electron microscope photomicrographs indicated grain size and grain distribution of monolithic ceramic at $\times 20 \mathrm{~K}$ magnification (A-I) and fractographic surface at $\times 60$ magnification (J-R) indicated fracture origin (red arrow), mist zone, hackle zone, and compression curl for of inCoris TZI (A-C and J-L), Vita Suprinity (D-F and $\mathbf{M}-\mathbf{O})$, IPS e.max CAD (G-I and P-R) upon slow (A, D, G and J, M, P), normal (B, E, $\mathbf{H}$ and $\mathbf{K}, \mathbf{N}, \mathbf{Q})$, and fast (C, F, I and $\mathbf{L}, \mathbf{O}, \mathbf{R})$ thermal tempering processes. 
of 23.96, 24.53, 25.02, and 37.83 degrees while detecting lithium metasilicate at $2 \theta$ angle of 38.37 degrees and also detecting lithium orthophosphate at $2 \theta$ angle of $22.48,23.28$, and 34.01 degrees.

\section{Discussion}

The modification of sintering parameters for enhancing mechanical properties of monolithic ceramic materials were previously reported on altering heating rate and sintering time, but no research has previously been investigated about cooling phase under the modification of thermal tempering processes, especially for Y-TZP, ZLS, and $\mathrm{LS}_{2}$. The results indicated significant different flexural strength of different monolithic ceramic materials at various thermal tempering processes and their interactions. Therefore, the null hypotheses were denied.

The material types affect the mechanical properties since they are composed of different components. The inCoris TZI is a Y-TZP, consisting entirely of crystalline content, as the crystalline content gives an advantage in terms of strength. The zirconia showed the highest flexural strength compared with the others. This was supported by the crack propagation of materials through the occurrence of $t$ - to m-phase transformation. Vita Suprinity is mainly composed of crystalline structures: lithium oxide and zirconia in a silicon dioxide glassy content. Zirconia was added to this material at a level of 8 to $12 \%$, giving so-called ZLS glass ceramic. Although the purpose of adding zirconia to $\mathrm{LS}_{2}$ glass ceramic is to enhance strength through dispersion strengthening, the flexural strength of this material is still lower than the E-groups. This was supported by the XRD, as the low conversion from lithium metasilicate to $\mathrm{LS}_{2}$ of $\mathrm{V}$-ceramic, compared with that of E-ceramic so that V-ceramic showed less flexural strength. Adding more zirconia was proved to hamper crystal growth since it increased the viscosity of glass and impeded the mobility of ions and thus the rate of solid-state reactions of crystal phase precipitation reduced. ${ }^{29}$

The flexural strength of the I-ceramic was significantly reduced by increasing the cooling rate. The IF represented the lowest flexural strength compared with IS and IN. This seems to be supported by the microstructure: under SEM analysis, the largest average grain size was shown in IF group. The higher the grain size, the less the flexural strength. The enlarged grain size is related to the relative proportions of the $\mathrm{t}$ - and $\mathrm{m}$-phases of the zirconia: the fast-thermal tempering groups showed a larger percentage of $m$-phase due to the rapid induction of stress by rapid cooling, which facilitated the t- to m-phase transformation. The highest flexural strength was presented in IS group, but the strength values of the IS and IN groups were not statistically different. In the SEM analysis of the fracture view, the IF group showed more numerous porosities than the other groups and also presented numerous fracture lines from the surface to pores and between pores; thus, it is more likely to break easily and has lower flexural strength. For the V-group, VN showed lower flexural strength compared with the other protocols, but there was no significant difference between the groups. The microstructures of $\mathrm{LS}_{2}$ crystals predominately influence on strength of glass ceramics. The interlocking pattern of minute shuttle-space shaped crystalline structure of the glass matrix-as observed in the VS group-plays a significant role in impeding crack propagation, leading to a higher flexural strength compared with spherical grains. ${ }^{29}$ The SEM analysis of fractured specimens illustrated that surface defects such as pores were origins of cracks, and the pattern of cracks in all groups seemed to be similar, leading to no significant different flexural strength among groups. For the E-group, ES showed the highest flexural strength; however, no statistical difference among groups was indicated. The ES group showed larger and longer grains, as well as less porosity, than the other groups. This might because the slow thermal tempering induced complete grain growth, resulting in longer grains and more interlocking of grains. The fracture images of all the E-groups presented similar patterns of crack propagation, resulting in no significant difference between the groups. The Weibull modulus of EF was the highest, followed by ES and then VS. A higher $\mathrm{m}$-value indicates more reliability, and less variation from sample to sample. Besides the $\mathrm{m}$-value of $\mathrm{EF}$, the $\mathrm{m}$-values of all groups were reliable: they were within the acceptable range of dental ceramics as supported by other studies. ${ }^{17}$

The tempering protocol based on the manufacturers' recommendations is comparable with the normal tempering process in this study. Based on the result of this investigation, it clearly indicated that slow thermal tempering procedure of Y-TZP was capable of achieving higher flexural strength and was recommended from this study. On the other hand, either slow-, normal-, or fast-thermal tempering can be performed for both ZLS and $\mathrm{LS}_{2}$ without significant influence on their flexural strength. Yet, to minimize processing time upon sintering process, especially for chair-side sintering process, the study suggested a fast-thermal tempering for both ZLS and $\mathrm{LS}_{2}$. The study provided data by which clinicians may actually benefit from altering tempering processes.

\section{Conclusion}

This study indicated that thermal tempering differently affected to flexural strengths of the monolithic Y-TZP, ZLS, and $\mathrm{LS}_{2}$. Slow thermal tempering of monolithic Y-TZP resulted in enhancing flexural strength. Strengthening of ZLS and $\mathrm{LS}_{2}$ cannot be achieved through tempering process; thus, either slow-, normal-, or fast-tempering procedure can be performed. Nevertheless, to minimize sintering time, rapid thermal tempering is more preferable for both ZLS and $\mathrm{LS}_{2}$.

\section{Funding}

This study received a grant from the Faculty of Dentistry, Khon Kean University, Ministry of Higher Education, Science, Research and Innovation, Royal Thai Government.

\section{Conflict of Interest}

None declared. 


\section{References}

1 Sen N, Sermet IB, Cinar S. Effect of coloring and sintering on the translucency and biaxial strength of monolithic zirconia. J Prosthet Dent 2018;119(2):308.e1-308.e7

2 Conrad HJ, Seong WJ, Pesun IJ. Current ceramic materials and systems with clinical recommendations: a systematic review. J Prosthet Dent 2007;98(5):389-404

3 Denry I, Holloway JA. Ceramics for dental applications: a review. Materials (Basel) 2010;3(1):351-368

4 Hallmann L, Ulmer P, Kern M. Effect of microstructure on the mechanical properties of lithium disilicate glass-ceramics. J Mech Behav Biomed Mater 2018;82:355-370

5 Gallina BL, Busato MCA, Sicoli EA, Camilotti V, Mendonca MJ. Aged translucent aesthetic zirconia: bond strength analysis. Eur J Dent 2019;13(1):5-10

6 Bachhav VC, Aras MA. Zirconia-based fixed partial dentures: a clinical review. Quintessence Int 2011;42(2):173-182

7 Piconi C, Maccauro G. Zirconia as a ceramic biomaterial. Biomaterials 1999;20(1):1-25

8 Denry I, Kelly JR. Emerging ceramic-based materials for dentistry. J Dent Res 2014;93(12):1235-1242

9 Kohorst P, Junghanns J, Dittmer MP, Borchers L, Stiesch M. Different CAD/CAM-processing routes for zirconia restorations: influence on fitting accuracy. Clin Oral Investig 2011;15(4):527-536

10 Matsui K, Yoshida H, Ikuhara Y. Isothermal sintering effects on phase separation and grain growth in yttria-stabilized tetragonal zirconia polycrystal. J Am Ceram Soc 2009;92(2):467-475

11 Sailer I, Fehér A, Filser F, Gauckler LJ, Lüthy H, Hämmerle CH. Five-year clinical results of zirconia frameworks for posterior fixed partial dentures. Int J Prosthodont 2007;20(4):383-388

12 Edelhoff D, Florian B, Florian W, Johnen C. HIP zirconia fixed partial dentures-clinical results after 3 years of clinical service. Quintessence Int 2008;39(6):459-471

13 Schmitt J, Holst S, Wichmann M, Reich S, Gollner M, Hamel J. Zirconia posterior fixed partial dentures: a prospective clinical 3-year follow-up. Int J Prosthodont 2009;22(6):597-603

14 Özkurt-Kayahan Z. Monolithic zirconia: a review of the literature. Biomed Res 2016;27(4):1427-1436

15 Guazzato M, Albakry M, Ringer SP, Swain MV. Strength, fracture toughness and microstructure of a selection of all-ceramic materials. Part II. Zirconia-based dental ceramics. Dent Mater 2004;20(5):449-456

16 Wang L, D’Alpino PH, Lopes LG, Pereira JC. Mechanical properties of dental restorative materials: relative contribution of laboratory tests. J Appl Oral Sci 2003;11(3):162-167
17 Bona AD, Anusavice KJ, DeHoff PH. Weibull analysis and flexural strength of hot-pressed core and veneered ceramic structures. Dent Mater 2003;19(7):662-669

18 Denry I, Kelly JR. State of the art of zirconia for dental applications. Dent Mater 2008;24(3):299-307

19 Marinis A, Aquilino SA, Lund PS, et al. Fracture toughness of yttria-stabilized zirconia sintered in conventional and microwave ovens. J Prosthet Dent 2013;109(3):165-171

20 Zhang Y, Lee JJ, Srikanth R, Lawn BR. Edge chipping and flexural resistance of monolithic ceramics. Dent Mater 2013; 29(12):1201-1208

21 Heffernan MJ, Aquilino SA, Diaz-Arnold AM. Haselton DR, Stanford CM, Vargas MA. Relative translucency of six all-ceramic systems. Part I: core materials. J Prosthet Dent 2002; 88(1):4-9

22 KellyJR, Nishimura I, Campbell SD. Ceramics in dentistry: historical roots and current perspectives. J Prosthet Dent 1996;75(1): 18-32

23 Chen YM, Smales RJ, Yip KH, Sung WJ. Translucency and biaxial flexural strength of four ceramic core materials. Dent Mater 2008;24(11):1506-1511

24 Stawarczyk B, Ozcan M, Hallmann L, Ender A, Mehl A, Hämmerlet $\mathrm{CH}$. The effect of zirconia sintering temperature on flexural strength, grain size, and contrast ratio. Clin Oral Investig 2013;17(1):269-274

25 Jiang L, Liao Y, Wan Q, Li W. Effects of sintering temperature and particle size on the translucency of zirconium dioxide dental ceramic. J Mater Sci Mater Med 2011;22(11):2429-2435

26 Ersoy NM, Aydoğdu HM, Değirmenci BU, Çökük N, Sevimay M. The effects of sintering temperature and duration on the flexural strength and grain size of zirconia. Acta Biomater Odontol Scand 2015;1(2-4):43-50

27 Juntavee N, Attashu S. Effect of sintering process on color parameters of nano-sized yttria partially stabilized tetragonal monolithic zirconia. J Clin Exp Dent 2018;10(8):e794-e804

28 Stefanic G, Grzeta B, Popović S, Musić S. In situ phase analysis of the thermal decomposition products of zirconium salts. Croat Chem Acta 1999;72(2-3):395-412

29 Thieme K, Avramov I, Rüssel C. The mechanism of deceleration of nucleation and crystal growth by the small addition of transition metals to lithium disilicate glasses. Sci Rep 2016;6:25451

30 Goharian P, Nemati A, Shabanian M, Afshar A. Properties, crystallization mechanism and microstructure of lithium disilicate glass-ceramic. J Non-Cryst Solids 2010;356(4):208-214 